\title{
Aplicação do gerenciamento de projetos no processo de desenvolvimento de novos produtos - um caso exploratório
}

\author{
Leandro Faria Almeida \\ Escola Politécnica da USP \\ almeida.leandro@hotmail.com
}

Paulo A. Cauchick Miguel, Dr.

Escola Politécnica da USP

paulo.miguel@poli.usp.br

\author{
Marly Monteiro de Carvalho, Dr. \\ Escola Politécnica da USP \\ marlymc@usp.br
}

Projetos têm sido freqüentemente utilizados como forma de realização do plano estratégico das empresas. Considerando que o desenvolvimento de novos produtos pode ser caracterizado como um projeto, o uso de metodologias, técnicas e ferramentas que auxiliem o seu gerenciamento é crucial. Nesse sentido, esse trabalho tem como objetivo apresentar uma visão geral da aplicação das práticas de gerenciamento de projetos no processo de desenvolvimento de novos produtos (PDP) através da análise dessas metodologias sugerindo uma forma de abordar e entender a dinâmica desses processos. Como resultado da análise teórica, o artigo apresenta uma referência cruzada entre o processo de desenvolvimento e de gerenciamento de projetos e também um estudo de caso. O estudo de caso consistiu da análise da utilização dessas práticas no processo de desenvolvimento de produtos de duas unidades de uma empresa do setor aeroespacial e conclui que, apesar de estarem bastante difundidas, as práticas de gerenciamento de projetos não estão sistematizadas no processo de desenvolvimento das unidades estudadas.

Palavras-chave: desenvolvimento de produtos, gerenciamento de projeto, ferramentas e técnicas

Projects have been often utilized as a means of achieving an organization's strategic plan. Considering that new product development can be characterized as a project, the usage of methodologies, techniques and tools to help its management is crucial. In this sense this paper aims at giving an overview of the application of project management practices in the product development process (PDP) through the analyses of these methodologies suggesting a way of looking at and understanding the dynamics of this process. As a result of the theoretical analysis the paper cross-refers new product development process and project management and presents a case study. The case study consisted of an analysis of the utilization of these practices in the product development process of two different units of an industry in the aerospace sector. The paper concludes that despite being spread, project management practices are not systematically applied in the development process of the unities studied.

Keywords: product development, project management, tools and techniques

\section{Introdução}

No ambiente turbulento de negócios, caracterizado por rápidas e intermitentes mudanças, as empresas têm passado por um processo de transformação, reorganizando-se para se tornarem mais efetivas e ágeis nas respostas aos problemas impostos por esse ambiente (PATAH; CARVALHO, 2005). Durante os últimos 5 a 10 anos, testemunha-se um aumento constante e significativo na dimensão com 
que as empresas adotam e se apóiam no gerenciamento de projetos para assegurar vantagens competitivas (GRANT; PENNYPACKER, 2006), sendo os projetos uma das características mais significativas das organizações contemporâneas (ENGWALL et al 2005). Segundo o PMI (2004), projetos são freqüentemente implementados como meios de realizar o plano estratégico da organização.

Por sua vez, de acordo com Chen et al. (2003), devido às rápidas mudanças tecnológicas, à globalização dos mercados, e à elevada concorrência dentro e fora do país, o desenvolvimento efetivo de produtos está surgindo como o maior foco de competição e iniciativa estratégica das empresas. Um produto de sucesso e lucrativo é o fator ganhador decisivo num ambiente como esse.

Cooper e Kleinschimdt (2001) afirma que o gerenciamento do desenvolvimento de produtos é de tamanha importância, que representa um processo que separa vencedores de perdedores (COOPER, KLEINCSHIMDT, 2001). O desenvolvimento de produto pode ser definido como um processo em que o produto é concebido, projetado e lançado no mercado e também inclui o feedback tanto da produção, como do uso do produto (ULRICH; EPPINGER, 1995). Uma elaboração adequada do processo de planejamento e desenvolvimento de novos produtos é muito importante e vital para as empresas, pois, permite conquistar novos mercados e atrair novos clientes (FORMAGGIO; MIGUEL, 2002). Porém, segundo Kerzner (2001), uma das tarefas mais difíceis em uma organização é o gerenciamento das atividades de desenvolvimento de novos produtos.

O processo de desenvolvimento de produtos (PDP) apresenta diversas características que o diferenciam de outros processos, tais como: elevado grau de incerteza e riscos das atividades e resultados; dificuldade de mudar as decisões iniciais; as atividades básicas seguem um ciclo iterativo; manipulação e geração de alto volume de informações; multiplicidade de requisitos a serem atendidos pelo processo (ROZENFELD et al., 2006), entre outros.

Além da complexidade técnica e da característica iterativa das atividades do desenvolvimento de novos produtos, é necessário gerenciar as interações entre várias disciplinas de engenharia, que podem estar alocadas em diferentes localidades, envolver uma centena de engenheiros e um grande número de subprojetos (CHEN et al. 2003; SÖDERLUND, 2002). A necessidade de se atingir tanto os prazos finais, como a integração dessas várias áreas de conhecimento vem ao encontro dos objetivos do gerenciamento de projetos (SÖDERLUND, 2002). Segundo o PMBoK (PMI, 2004), o gerenciamento de projetos é a aplicação de conhecimentos, habilidades, ferramentas e técnicas para desenvolver atividades que visam atingir os requisitos do projeto.

Projetos têm sido discutidos como mecanismos de integração, possibilitando uma integração multifuncional (ENGWALL et al., 2005) e, dentre outras coisas, como uma ferramenta efetiva na organização do desenvolvimento de produto (ENGWALL et al., 2005).

Nesse contexto, o presente artigo tem como objetivo analisar como pode ser realizado o gerenciamento das atividades de desenvolvimento de produto, utilizando as práticas de gerenciamento de projeto. Em outras palavras, o objetivo é entender como a metodologia de gerenciamento de projetos se aplica ao processo de desenvolvimento de um novo produto.

\section{Referencial Teórico}

O desenvolvimento de produtos compreende as atividades através das quais a empresa irá conceber o produto, desenvolver as especificações técnicas do produto e do processo de fabricação e comercializá-lo. A necessidade de desenvolvimento desse produto pode surgir dos planos estratégicos da empresa, e deverá ser fortemente direcionado pelas necessidades do mercado e pelo ambiente tecnológico. O PDP caracteriza-se como um processo complexo e de elevada incerteza onde, além de apresentar inúmeras atividades interrelacionadas e interfaces técnicas, deve vincular seus processos e atividades aos diversos requisitos de mercado, aos requisitos internos da empresa e das demais partes da cadeia produtiva. 
Além disso, o processo de desenvolvimento de produtos (PDP) se utiliza de uma variedade de ferramentas e técnicas que auxiliam no desenvolvimento e nas especificações técnicas do produto. Essas ferramentas, de maneira geral, são incluídas nos modelos de desenvolvimento de produtos que, além de sugerir a aplicação dessas ferramentas em momentos específicos do projeto, buscam "descrever as atividades, recursos, informações, fases, responsabilidades e outras possíveis dimensões do processo" (ROZENFELD et al., 2006).

Vários autores têm trabalho no desenvolvimento de modelos para o desenvolvimento de produto. De acordo com Formoso et al. (2002), alguns modelos tentam simplesmente descrever o processo, outros focam no processo de desenvolvimento como um todo, enquanto outros tratam do projeto do produto em si. Ainda de acordo com esses autores, existem também modelos que buscam fornecer métodos e ferramentas para suportar o gerenciamento do desenvolvimento de produto.

Como referência para análise do processo de desenvolvimento de produto (PDP) no presente trabalho é considerado o modelo proposto por Rozenfeld et al. (2006), ilustrado resumidamente na figura 1 . O modelo foi selecionado por ser um modelo "voltado para empresas de manufatura de bens de consumo duráveis e de capital, com ênfase na tecnologia mecânica de fabricação" (ROZENFELD et al., 2006), sendo uma referência adequada para o estudo de caso a ser realizado. Deve também ser mencionado que a maioria dos autores concorda em grande parte sobre as etapas básicas do processo de desenvolvimento e, para os objetivos do trabalho em questão, as diferenças entre um modelo e outro não impactariam de forma significativa os resultados do trabalho.

\begin{tabular}{|c|c|c|c|}
\hline MACROFASES & PRÉ-DESENVOLVIMENTO & DESENVOLVIMENTO & PÓS-DESENVOLVIMENTO \\
\hline \multirow{4}{*}{ FASES } & $\begin{array}{c}\text { Planejamento Estratégico } \\
\text { de Produtos }\end{array}$ & Projeto Informacional & $\begin{array}{c}\text { Acompanhar Produto e } \\
\text { Processo }\end{array}$ \\
\cline { 2 - 3 } & Planejamento do Projeto & Projeto Conceitual & $\begin{array}{c}\text { Descontinuar Produto no } \\
\text { Mercado }\end{array}$ \\
\cline { 2 - 3 } & & Projeto Detalhado & \\
\cline { 3 - 3 } & & $\begin{array}{c}\text { Preparação da Produção } \\
\text { do Produto }\end{array}$ \\
\cline { 2 - 3 } & Lançamento do Produto & \\
\hline
\end{tabular}

Figura 1 Macro-fases e Fases do Desenvolvimento de Produtos Fonte: Adaptado de Rozenfeld et al. (2006)

A seguir são listados resumidamente os principais resultados de cada fase, de acordo com Rozenfeld et al. (2006). A fase de Planejamento Estratégico resultará em dois documentos, que são o portfolio de produtos contendo a descrição e data de início de desenvolvimento e lançamento de cada produto e a minuta de projeto, que é o primeiro documento que diz respeito a um projeto específico. Na fase de Planejamento do Projeto, a minuta do projeto será utilizada para desenvolver um plano detalhado do projeto, com prazos, custos, atividades, etc.

$\mathrm{Na}$ fase seguinte, Projeto Informacional, são geradas as especificações meta do produto, composta pelos requisitos e informações qualitativas sobre o produto. No Projeto Conceitual, são geradas e estudadas soluções que atendam as especificações meta. Na fase de Projeto Detalhado é realizado o detalhamento da concepção do produto (solução escolhida), gerando suas especificações finais. $\mathrm{Na}$ fase de Preparação para Produção do Produto são realizadas as atividades definição e homologação dos processos de fabricação. Em seguida, o produto é então lançado no mercado.

Para análise das metodologias de gerenciamento de projeto, foi considerada a estrutura proposta no PMBoK, pelo PMI (2004). O PMI (2004) sugere que o gerenciamento de projeto é realizado por meio da aplicação e integração dos seguintes processos: iniciação, planejamento, execução, monitoramento e controle, e encerramento. Esses processos são organizados nas áreas de conhecimento de integração, escopo, tempo, custo, qualidade, recursos humanos, comunicação, riscos e aquisição. Para cada área de conhecimento são apresentados processos específicos, divididos de acordo com o grupo de processos, com o objetivo de orientar a aplicação dos conhecimentos e habilidades de gerenciamento 
do projeto durante o projeto. Para cada processo são listadas as entradas necessárias para desenvolvimento do processo, as ferramentas e técnicas aplicáveis, e as saídas (resultados/produtos do processo). A seguir é dada uma breve descrição de cada grupo de processo.

Segundo o PMI (2004), os processos de iniciação são freqüentemente realizados fora do escopo de controle do projeto pela organização ou pelos processos de programa ou de portfolio. Os processos de iniciação definem e autorizam o início de um projeto, onde são apresentados os objetivos do projeto, uma descrição básica do escopo, das entregas, da duração, uma previsão dos recursos, as premissas e as restrições.

Os processos do grupo de planejamento geralmente envolvem todas as áreas de conhecimento. É durante esse processo que serão definidos mais claramente os objetivos do projeto e planejadas as ações necessárias para que esses objetivos sejam alcançados. Segundo o PMI (2004), a fase de planejamento geralmente não está incluída no escopo do projeto.A principal saída dessa fase é o plano de gerenciamento do projeto, que integra todos os planos auxiliares em um só plano e define como o projeto é executado, monitorado, controlado e encerrado (PMI, 2004).

De acordo com o PMBoK (PMI, 2004), os processos de execução envolvem a coordenação das pessoas e recursos, além da integração e da realização das atividades do projeto planejadas anteriormente.

O objetivo dos processos de monitoramento e controle é observar e medir o desempenho do projeto com relação ao que foi definido no plano de gerenciamento do projeto. O monitoramento e controle do projeto fornece feedback para as atualizações do plano de gerenciamento do projeto no início de cada fase.

O grupo de processo de encerramento formaliza o encerramento do projeto ou fase do projeto. A Figura 2 mostra as áreas que apresentam processos relacionados, separados nos grupos de processos, de acordo com o PMBoK (PMI, 2004).

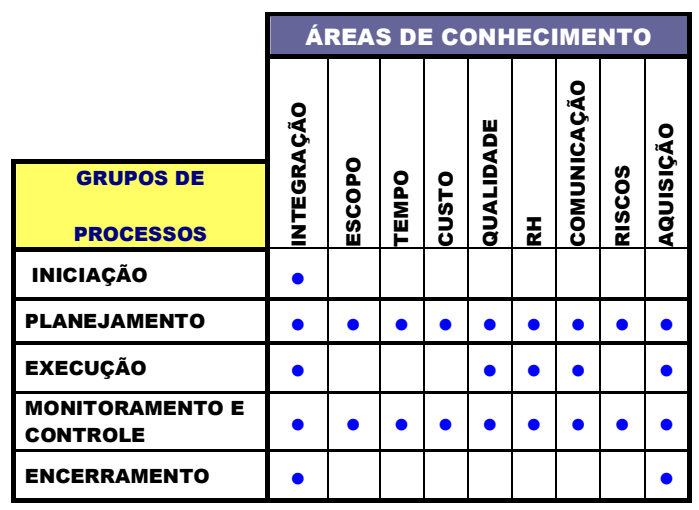

Legenda: • - existem processos relacionados.

Figura 2 Grupos de processos e Áreas de Conhecimento em Gerenciamento de projetos

Os modelos de desenvolvimentos de produto são geralmente divididos em fases e, segundo o PMI (2004), quando isso acontece, os grupos de processos de gerenciamento de projetos normalmente se repetem dentro de cada fase, durante toda a vida do projeto, conforme ilustrado na

Figura 3. 


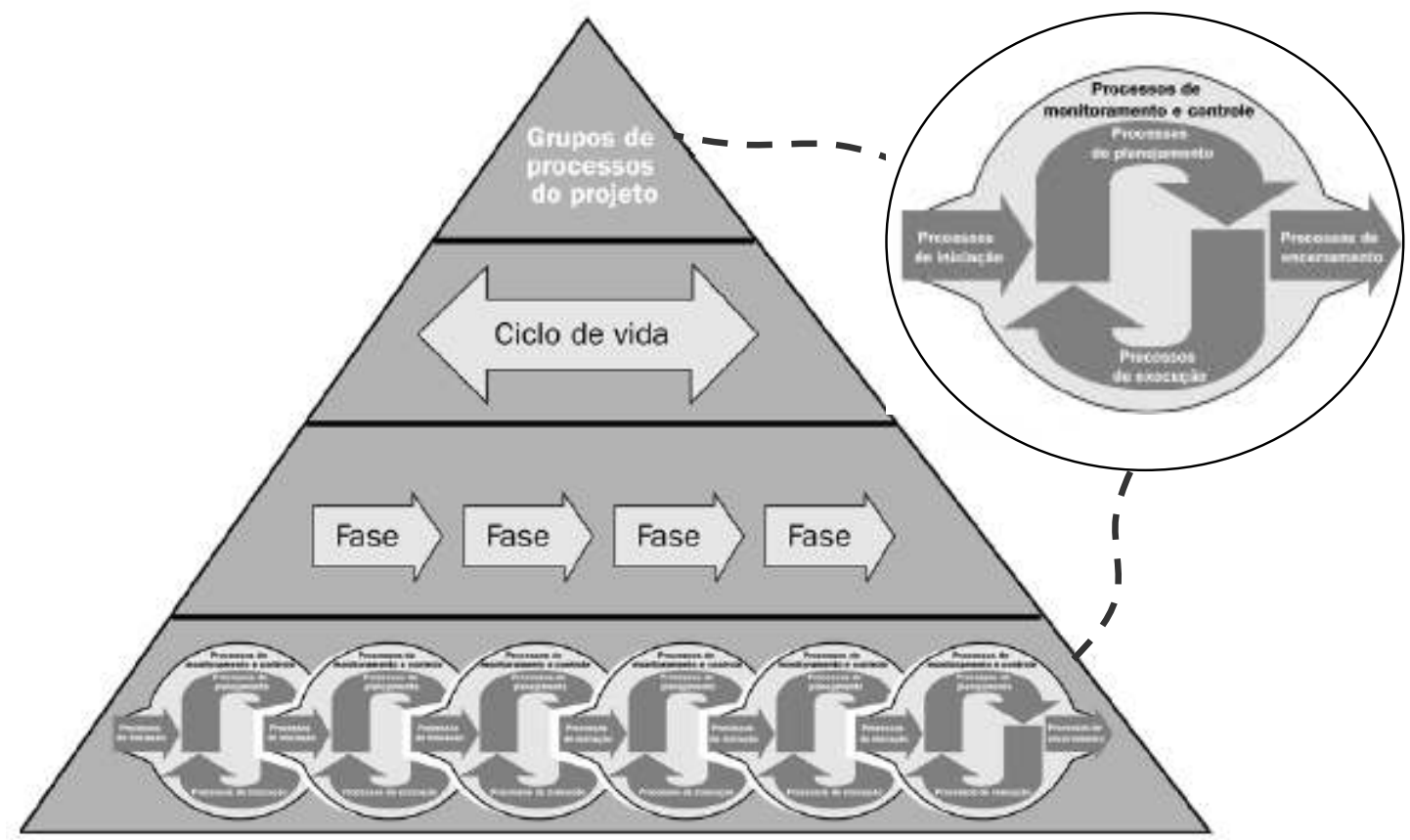

Figura 3 Triângulo do grupo de processos de gerenciamento de projetos (adaptado de PMI, 2004)

\section{Métodos e técnicas de pesquisa}

O presente trabalho pode ser caracterizado como uma pesquisa exploratória, por se tratar de um trabalho onde se busca definir melhor o problema e obter insights sobre o assunto. O método de pesquisa utilizado no desenvolvimento da pesquisa foi o estudo de caso, uma vez que o objetivo era verificar no campo empírico se as ferramentas e técnicas analisadas nesse artigo eram utilizadas no gerenciamento do processo de desenvolvimento. Dessa forma, o método se justifica pelo fato do pesquisador não apresentar controle sobre os eventos, e o foco estar em eventos contemporâneos, conforme propõe Yin (1984).

A análise da aplicação da abordagem de gerenciamento de projetos no processo de desenvolvimento de produtos realizada neste trabalho se restringiu às atividades contidas na macrofase de desenvolvimento Figura 1, também denominada de projeto do produto. É durante essa macrofase que ocorrem as definições do produto em si; as especificações técnicas, o seu processo de fabricação, a forma construtiva, etc. (ROZENFELD et al., 2006).

Para elaboração do trabalho, primeiramente procurou-se estabelecer uma relação entre as metodologias de gerenciamento de projeto e o processo de desenvolvimento de produto. Para isso, as atividades das fases de desenvolvimento de produtos foram agrupadas nos grupos de processos (iniciação, planejamento, execução, monitoramento e controle, e encerramento) propostos no PMBoK, (PMI, 2004). Isso foi feito identificando-se as características de cada atividade do desenvolvimento do produto. Levando em consideração essas características e o que foi mostrado anteriormente na Figura 3 , que para um projeto que é dividido em fases as atividades de gerenciamento de projeto se repetem. As atividades de desenvolvimento de produto foram então classificadas e agrupadas dentro do grupo de processo a que estariam mais relacionadas. O resultado dessa primeira análise é mostrado na Figura 4.

Foi então realizada uma análise da aplicação das ferramentas e técnicas de gerenciamento de projeto propostas para cada processo do PMBoK (PMI, 2004) no processo de desenvolvimento de produto, 
sendo utilizada a Figura 4 para orientar essas análises. Essa análise foi descrita por grupo de processo e, dentro de cada grupo, se refere às atividades de desenvolvimento de produto. Outro resultado foi a seleção das ferramentas e técnicas apresentadas no PMBoK (PMI, 2004) para posterior verificação de sua aplicação nas unidades estudadas. Os critérios para a seleção foram: ferramentas cuja definição seja clara o bastante e/ou que poderiam ser aplicadas de forma objetiva. Por exemplo: uma ferramenta como "habilidades de comunicação" não poderia ser aplicada objetivamente, pois requereria o desenvolvimento de habilidades pessoais para implementá-la e é de certa forma subjetiva, enquanto que "métodos de distribuição das informações" são mais facilmente entendidos e poderiam ser implantados definindo-se uma sistemática para comunicação. Foram também agrupadas algumas ferramentas quando o método utilizado não interessava para a análise. Por exemplo, as estimativas de custo paramétrica, "bottom-up" e análoga foram agrupadas em uma única ferramenta de estimativa de custo. Essas ferramentas são apresentadas na Tabela 1, nas colunas de ferramentas de gerenciamento de projetos.

O estudo de caso foi conduzido em duas unidades de uma empresa nacional de grande porte do setor aeroespacial que desenvolve, fabrica e comercializa aviões. Os projetos desenvolvidos nas unidades estudadas podem ser classificados como projetos incrementais, que são projetos derivados ou com pequenas modificações com relação aos projetos já existentes, conforme define Rozenfeld et al. (2006). Em média, cada gerente de projetos das unidades estudadas gerencia dez projetos/ano (paralelamente), com uma duração média de um ano cada.

Para realização do estudo, foram feitas análises documentais de projetos desenvolvidos no último ano nas unidades em questão e também realizadas entrevistas semi-estruturadas com os gerentes de projetos. Para tal, utilizou-se de um questionário que continha as ferramentas que são apresentadas na tabela 1 com o objetivo de identificar se elas eram aplicadas no processo de desenvolvimento das unidades ou não, e se eram aplicadas de maneira formal ou informal. A aplicação informal foi definida como sendo aquela realizada sem que houvesse documentação ou aquela realizada esporadicamente sem que fizesse parte de uma metodologia comum de gerenciamento de projetos da unidade. $\mathrm{O}$ questionário foi validado previamente com os gerentes de projeto das próprias unidades, sendo a entrevista realizada apenas com um gerente de projetos de cada unidade. Os gerentes de projeto também foram questionados sobre a importância da aplicação de cada ferramenta para que se obtenha um bom desempenho do projeto. Essa avaliação foi feita atribuindo-se um critério de 1 a 5, onde "1menos importante", "2-pouco importante"; "3-média importância", "4-importante" e "5-muito importante".

\section{Resultados da Análise Teórica}

Nesta seção é apresentada a síntese da análise teórica das metodologias de gerenciamento de projeto e o processo de desenvolvimento de produto, tendo como base as referências PMI (2004) e Rozenfeld et al. (2006), respectivamente.

A Figura 4 apresenta o resultado do agrupamento das atividades de desenvolvimento do produto dentro dos grupos de processo de gerenciamento de projetos. Uma primeira implicação que essa divisão pode trazer é que as atividades que se encontram no grupo de execução são aqueles centrais do processo de desenvolvimento, ou seja, são atividades através das quais o produto será realmente criado. Sendo assim, pode-se dizer que as atividades contidas nos grupos de iniciação, planejamento, monitoramento e controle, e encerramento, deverão estar voltadas para suportar as atividades do grupo de execução. As análises que são apresentadas a seguir derivam dessas implicações.

A seguir, passa-se a discriminar as principais conclusões da análise teórica segundo os grupos de processos de gerenciamento de projet 
Tabela 1 - Ferramentas de Gerenciamento de projeto nas atividades de desenvolvimento de produto.

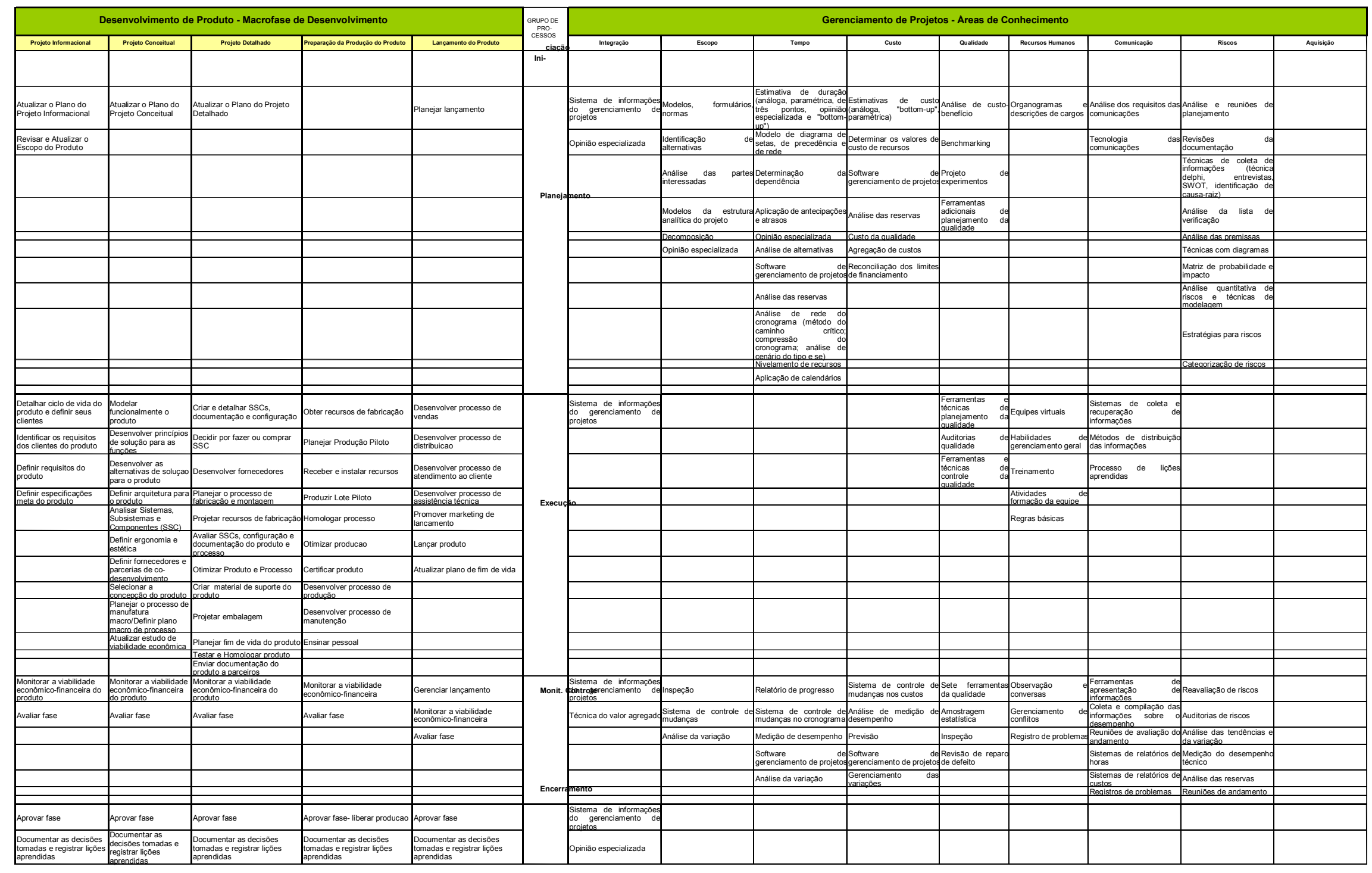




\begin{tabular}{|c|c|c|c|c|c|}
\hline \multirow{3}{*}{$\begin{array}{c}\text { MACRO FASE -> } \\
\text { FASE -> } \\
\text { GRUPODE } \\
\text { PROCESSO } \\
\end{array}$} & \multicolumn{5}{|c|}{ DESENVOLVIMENTO } \\
\hline & \multirow{2}{*}{$\begin{array}{c}\text { Projeto } \\
\text { Informacional }\end{array}$} & \multirow{2}{*}{ Projeto Conceitual } & \multirow{2}{*}{ Projeto Detalhado } & \multirow{2}{*}{$\begin{array}{l}\text { Preparação da } \\
\text { Produção do } \\
\text { Produto } \\
\end{array}$} & \multirow{2}{*}{ Lançamento do Produto } \\
\hline & & & & & \\
\hline INICIAÇÃO & - & - & - & - & - \\
\hline \multirow{2}{*}{ PLANEJAMENTO } & $\begin{array}{l}\text { Atualizar o Plano do } \\
\text { Projeto Informacional }\end{array}$ & $\begin{array}{l}\text { Atualizar o Plano do } \\
\text { Projeto Conceitual }\end{array}$ & $\begin{array}{l}\text { Atualizar o Plano do } \\
\text { Projeto Detalhado }\end{array}$ & & Planejar lançamento \\
\hline & $\begin{array}{l}\text { Revisar e Atualizar o } \\
\text { Escopo do Produto }\end{array}$ & & & & \\
\hline \multirow{12}{*}{ EXECUÇÃO } & $\begin{array}{l}\text { Detalhar ciclo de vida } \\
\text { do produto e definir } \\
\text { seus clientes }\end{array}$ & $\begin{array}{c}\text { Modelar } \\
\text { funcionalmente o } \\
\text { produto }\end{array}$ & $\begin{array}{l}\text { Criar e detalhar SSCs, } \\
\text { documentação e } \\
\text { configuração }\end{array}$ & $\begin{array}{l}\text { Obter recursos de } \\
\text { fabricação }\end{array}$ & Desenvolver processo de vendas \\
\hline & $\begin{array}{l}\text { Identificar os requisitos } \\
\text { dos clientes do produto }\end{array}$ & $\begin{array}{l}\text { Desenvolver princípios } \\
\text { de solução para as } \\
\text { funçōes }\end{array}$ & $\begin{array}{l}\text { Decidir por fazer ou } \\
\text { comprar SSC }\end{array}$ & $\begin{array}{l}\text { Planejar Produção } \\
\text { Piloto }\end{array}$ & Desenvolver processo de distribuição \\
\hline & $\begin{array}{l}\text { Definir requisitos do } \\
\text { produto }\end{array}$ & $\begin{array}{l}\text { Desenvolver as } \\
\text { alternativas de solução } \\
\text { para o produto }\end{array}$ & $\begin{array}{l}\text { Desenvolver } \\
\text { fornecedores }\end{array}$ & $\begin{array}{l}\text { Receber e instalar } \\
\text { recursos }\end{array}$ & $\begin{array}{l}\text { Desenvolver processo de atendimento ao } \\
\text { cliente }\end{array}$ \\
\hline & $\begin{array}{c}\text { Definir especificações } \\
\text { meta do produto }\end{array}$ & $\begin{array}{c}\text { Definir arquitetura para } \\
\text { o produto }\end{array}$ & $\begin{array}{l}\text { Planejar o processo de } \\
\text { fabricação e montagem }\end{array}$ & Produzir Lote Piloto & $\begin{array}{c}\text { Desenvolver processo de assistência } \\
\text { técnica }\end{array}$ \\
\hline & & $\begin{array}{c}\text { Analisar Sistemas, } \\
\text { Subsistemas e } \\
\text { Componentes (SSC) } \\
\end{array}$ & $\begin{array}{c}\text { Projetar recursos de } \\
\text { fabricação }\end{array}$ & Homologar processo & Promover marketing de lançamento \\
\hline & & $\begin{array}{c}\text { Definir ergonomia e } \\
\text { estética }\end{array}$ & $\begin{array}{c}\text { Avaliar SSCs, } \\
\text { configuração e } \\
\text { documentação do } \\
\text { produto e processo }\end{array}$ & Otimizar produção & Lançar produto \\
\hline & & $\begin{array}{l}\text { Definir fornecedores e } \\
\text { parcerias de co- } \\
\text { desenvolvimento }\end{array}$ & $\begin{array}{l}\text { Otimizar Produto e } \\
\text { Processo }\end{array}$ & Certificar produto & Gerenciar lançamento \\
\hline & & $\begin{array}{c}\text { Selecionar a } \\
\text { concepção do produto }\end{array}$ & $\begin{array}{l}\text { Criar material de } \\
\text { suporte do produto }\end{array}$ & $\begin{array}{c}\text { Desenvolver processo } \\
\text { de produção }\end{array}$ & Atualizar plano de fim de vida \\
\hline & & $\begin{array}{l}\text { Planejar o processo de } \\
\text { manufatura } \\
\text { macro/Definir plano } \\
\text { macro de processo }\end{array}$ & Projetar embalagem & $\begin{array}{l}\text { Desenvolver processo } \\
\text { de manutenção }\end{array}$ & \\
\hline & & $\begin{array}{c}\text { Atualizar estudo de } \\
\text { viabilidade econômica }\end{array}$ & $\begin{array}{c}\text { Planejar fim de vida do } \\
\text { produto }\end{array}$ & Ensinar pessoal & \\
\hline & & & $\begin{array}{c}\text { Testar e Homologar } \\
\text { produto }\end{array}$ & & \\
\hline & & & $\begin{array}{l}\text { Enviar documentação } \\
\text { do produto a parceiros }\end{array}$ & & \\
\hline \multirow[t]{2}{*}{$\begin{array}{l}\text { MONITORAMENTO } \\
\text { E CONTROLE }\end{array}$} & $\begin{array}{l}\text { Monitorar a viabilidade } \\
\text { econômico-financeira } \\
\text { do produto }\end{array}$ & $\begin{array}{l}\text { Monitorar a viabilidade } \\
\text { econômico-financeira } \\
\text { do produto }\end{array}$ & $\begin{array}{l}\text { Monitorar a viabilidade } \\
\text { econômico-financeira } \\
\text { do produto }\end{array}$ & $\begin{array}{l}\text { Monitorar a viabilidade } \\
\text { econômico-financeira }\end{array}$ & $\begin{array}{l}\text { Monitorar a viabilidade econômico- } \\
\text { financeira }\end{array}$ \\
\hline & Avaliar fase & Avaliar fase & Avaliar fase & Avaliar fase & Avaliar fase \\
\hline \multirow[b]{2}{*}{ ENCERRAMENTO } & Aprovar fase & Aprovar fase & Aprovar fase & $\begin{array}{l}\text { Aprovar fase- liberar } \\
\text { produçăo }\end{array}$ & Aprovar fase \\
\hline & $\begin{array}{l}\text { Documentar as } \\
\text { decisōes tomadas e } \\
\text { registrar liçōes } \\
\text { aprendidas }\end{array}$ & $\begin{array}{l}\text { Documentar as } \\
\text { decisōes tomadas e } \\
\text { registrar liçōes } \\
\text { aprendidas }\end{array}$ & $\begin{array}{l}\text { Documentar as } \\
\text { decisozes tomadas e } \\
\text { registrar liçōes } \\
\text { aprendidas }\end{array}$ & $\begin{array}{l}\text { Documentar as } \\
\text { decisōes tomadas e } \\
\text { registrar liçōes } \\
\text { aprendidas }\end{array}$ & $\begin{array}{l}\text { Documentar as decisões tomadas e } \\
\text { registrar lições aprendidas }\end{array}$ \\
\hline
\end{tabular}

Figura 4 Atividades do PDP distribuídas nos grupos de processos

\subsection{Iniciação}

Não foi selecionada nenhuma ferramenta para esse grupo de processo, uma vez que os processos de iniciação são geralmente realizados fora do escopo do projeto e, para o trabalho em questão, estão sendo consideradas apenas as atividades contidas na macrofase de desenvolvimento. Analisando o modelo proposto por Rozenfeld et al. (2006) segundo a divisão proposta neste trabalho, as atividades e resultados do grupo de processo de iniciação aconteceriam na macrofase de pré-desenvolvimento, mais especificamente no planejamento do projeto. No entanto, destaca-se que estas duas saídas do grupo de processo de iniciação (termo de abertura do projeto e declaração do escopo preliminar do projeto) da metodologia de gerenciamento de projetos, são entradas importantes para a macrofase de desenvolvimento, foco deste artigo.

\subsection{Planejamento}

O modelo de Rozenfeld et al. (2006) propõe, no início de cada fase, a atualização do plano do projeto elaborado na macrofase de pré-desenvolvimento.

Algumas ferramentas citadas no PMBoK (PMI, 2004) para os grupos de planejamento e que são de grande importância para qualquer fase do desenvolvimento do produto, são as relacionadas às áreas de tempo e custo do projeto, tais como: estimativas de custo e duração, linha de base dos custos, análise de alternativas, nivelamento de recursos, análise de reservas, entre outras.

Das áreas de escopo e qualidade, práticas como análise das partes interessadas, opinião especializada, identificação de alternativas e benchmarking podem auxiliar na identificação dos interessados do projeto, na identificação de abordagens de trabalho diferentes e mais adequadas para um certo tipo de 
desenvolvimento, assim como estabelecer padrões para medição de desempenho e obter idéias de melhoria. $\mathrm{Na}$ fase de projeto informacional, por exemplo, os principais resultados esperados são os requisitos e informações qualitativas do produto, o que faz da identificação das partes interessadas uma ferramenta importante para que sejam consideradas as necessidades de todas as áreas envolvidas e afetadas.

A utilização de organogramas e descrição dos cargos pode auxiliar principalmente quando se trabalha com grupos multifuncionais e/ou em uma estrutura matricial.

É importante também que se defina para cada fase do desenvolvimento, o plano de gerenciamento de comunicação, para que se estabeleça a forma e freqüência de comunicação, os métodos utilizados, bem como "incluir diretrizes para reuniões de andamento de projeto" (PMI, 2004).

Outra área de grande importância a ser considerada é a de riscos, que através da utilização de ferramentas de levantamento e avaliação de riscos irá resultar em um plano de gerenciamento de riscos e no levantamento dos principais riscos do projeto. Dessa forma, conforme propõe Rozenfeld et al. (2006), deverão ser analisados os riscos associados à complexidade da tecnologia envolvida, à inabilidade e/ou inexperiência em gerenciar projetos de desenvolvimento de produtos e às prováveis mudanças em legislações e regulamentações. No início de cada fase do desenvolvimento, esses riscos devem ser reavaliados considerando-se as novas condições do projeto e o fato de que à medida que $o$ projeto avança, as incertezas diminuem.

\subsection{Execução}

Considerando que as atividades contidas nesse grupo consistem da execução do que foi planejado anteriormente, ferramentas das áreas de integração, comunicação e recursos humanos são muito importantes para um bom desempenho dessas atividades.

Algumas ferramentas e técnicas sugeridas para esse grupo incluem sistemas de coleta e recuperação de informações e modelos de distribuição das informações que definirão como as informações serão coletadas e recuperadas durante o desenvolvimento, bem como a sua distribuição e/ou disponibilização para as partes interessadas no momento oportuno. Visto que o processo de desenvolvimento de produtos geralmente ocorre de maneira iterativa e requer o envolvimento de diversas áreas, essas ferramentas são fundamentais para que o desenvolvimento ocorra de maneira eficaz.

A utilização de equipes virtuais, conforme sugere o PMBoK (PMI, 2004), pode ser necessária quando se têm equipes de desenvolvimento trabalhando em diferentes pontos geográficos. São também muito importantes as habilidades de gerenciamento geral nessa etapa, uma vez que irão tratar de aspectos que não estão diretamente ligados a características técnicas do produto, mas aos aspectos da equipe de desenvolvimento, como motivação, cooperação, conflitos, resolução de problemas, entre outros e que contribuem fortemente para o bom andamento do projeto.

\subsection{Monitoramento e Controle}

As ferramentas desse grupo de processo irão comparar o que foi executado com o que estava planejado, fazendo a verificação principalmente de quatro aspectos do projeto e do produto em si, que são: custo, prazo, qualidade e escopo. Sendo assim, as linhas de base de custo, qualidade, escopo e tempo devem ser monitoradas continuamente, garantindo que o produto irá chegar ao mercado em condições de competir e se estabelecer em meio a tantos outros produtos, muitas vezes pouco diferenciados. A técnica de valor agregado, conforme cita o PMBoK (PMI, 2004) pode fornecer uma medida do desempenho do projeto, bem como prever o desempenho futuro com base no desempenho passado. 
O prazo de lançamento do produto é fundamental, pois em certos casos, há o risco do produto já estar obsoleto no momento do lançamento. $\mathrm{O}$ atendimento aos prazos estabelecidos poderá representar também um fator de vantagem competitiva, garantindo que o produto seja lançado no tempo certo no mercado. Dessa forma, ferramentas como relatórios de progresso, medições de desempenho e análise da variação, entre outras, podem ser utilizadas para monitoramento desse parâmetro.

O controle da qualidade e do escopo irá garantir que as funcionalidades esperadas para aquele produto atendem os requisitos do mercado, assim como se o seu desempenho é satisfatório e o nível de qualidade compatível com o posicionamento que se espera do produto com relação a seus concorrentes. O PMBoK (PMI, 2004) sugere ferramentas como inspeção e sistema de gerenciamento de configuração, por exemplo, para auxiliar no controle do escopo do produto. Da mesma forma, é sugerida a inspeção para controle da qualidade do produto, assim como ferramentas para solução de problemas (diagrama de Pareto, histograma, fluxograma, diagrama de causa e efeito, etc.), e para o monitoramento do processo (gráfico de controle), que são importantes tanto na fase de desenvolvimento do produto e da preparação para produção e lançamento do produto.

Além desses quatro parâmetros citados acima, os riscos do projeto podem ser monitorados utilizandose ferramentas como reavaliação de riscos, análise de reservas, análise das tendências e da variação, reuniões de andamento, etc. Essas ferramentas permitirão saber se os riscos levantados em fases anteriores do desenvolvimento foram mitigados no decorrer do projeto, se as medidas de contingência estão adequadas e pode orientar na tomadas de decisão.

A principal saída deste grupo de processos é o plano do projeto, estratificado por áreas de conhecimento em gerenciamento de projetos. Já na metodologia de desenvolvimento de produtos, o plano é estratificado por fases: plano do projeto informacional, plano do projeto conceitual, plano do projeto detalhado e plano de lançamento.

\subsection{Encerramento}

Para essa etapa, o PMBoK sugere a aplicação de ferramentas e técnicas como metodologia de gerenciamento de projetos, sistema de informações do gerenciamento de projetos e opinião especializada, que de certa forma tem uma definição um pouco vaga. Porém, as práticas de gerenciamento de projeto deverão fornecer informação suficiente para que se decida pela aprovação ou não da fase de desenvolvimento e para que as decisões tomadas sejam registradas. Desta forma, o início da próxima fase seria formalmente autorizado.

As principais saídas deste grupo de processo são o encerramento administrativo e o encerramento de contrato, que assume especial importância quando o projeto tem um cliente externo. Destaca-se ainda a importância da aprovação das partes interessadas (stakeholders) e a discussão das lições aprendidas.

\section{Resultados do Estudo de Caso}

A seguir são apresentados os resultados das análises realizadas nas unidades de estudo sobre a utilização das práticas de gerenciamento de projeto no processo de desenvolvimento do produto.

Das ferramentas selecionadas no PMBoK (apresentadas na Tabela 1), em média 76\% são utilizadas no processo de desenvolvimento das unidades de estudo. Das ferramentas que são utilizadas, em média $44 \%$ (34\% do total de ferramentas) são utilizadas de maneira formal, enquanto que 56\% (43\% do total de ferramentas) são utilizadas de maneira informal.

Do total das ferramentas, $77 \%$ foi considerada de importância 4 ou 5, das quais $43 \%$ são utilizadas de maneira formal, $44 \%$ de maneira informal e $13 \%$ não são utilizadas.

Para as ferramentas consideradas formais que são utilizadas pela empresa, foi atribuído importância 4 ou 5 para $100 \%$ delas. Para as ferramentais informais, $79 \%$ foram consideradas de importância 4 ou 5 , enquanto que para as que não são utilizadas, $42 \%$ são de importância 4 ou 5. 
As Figuras 6 e 7 mostram como as ferramentas estão distribuídas nos grupos de processos e áreas de conhecimento.

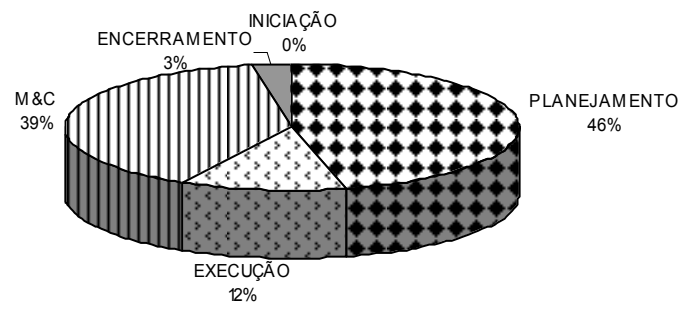

Figura 6: Ferramentas utilizadas por grupo de processo (\% do total de ferramentas)

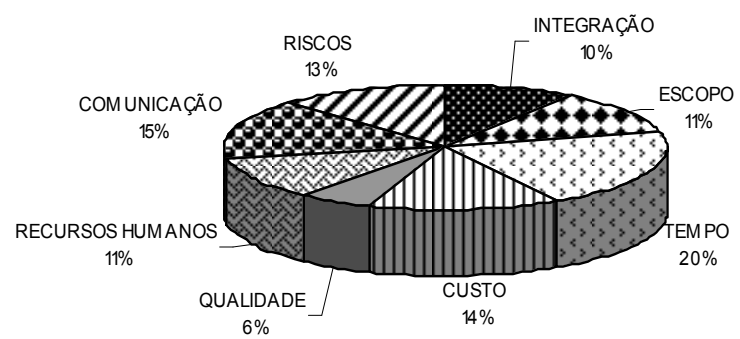

Figura 7: Ferramentas utilizadas por áreas de conhecimento (\% do total de ferramentas)

A Figura 6 mostra que o maior número de ferramentas utilizadas estão no grupo de processo de planejamento (46\%) e, em seguida, no grupo de monitoramento e controle (39\%). Já na Figura 7 pôde ser verificado que o maior número de ferramentas utilizadas está nas áreas de tempo $(20 \%)$, comunicação $(15 \%)$, custo $(14 \%)$ e riscos $(13 \%)$.

Alguns pontos que podem ser destacados na Figura 8 são: 1) o grupo de monitoramento é o que apresentou o maior número de ferramentas informais com importância 4 ou $5(88 \%)$, seguido do grupo de execução $(80 \%)$ e planejamento $(72 \%) ; 2)$ todas as ferramentas utilizadas formalmente no processo de desenvolvimento foram consideradas de importância 4 ou 5;3) o grupo de execução é o que apresentou o maior número de ferramentas (43\%) com importância 4 ou 5 que não são utilizadas no processo de desenvolvimento, seguido do grupo de planejamento (26\%) e de monitoramento e controle (21\%).

\begin{tabular}{|c|c|c|c|c|c|c|}
\hline \multirow[b]{2}{*}{$\begin{array}{l}\text { GRUPOS DE } \\
\text { PROCESSO } \\
\end{array}$} & \multicolumn{6}{|c|}{$\%$ DAS FERRAMENTAS UTILIZADAS } \\
\hline & 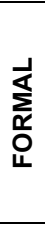 & 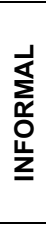 & 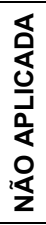 & 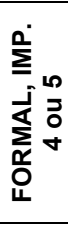 & 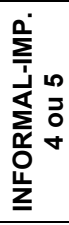 & 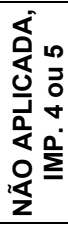 \\
\hline INICIAÇÃO & 0 & 0 & 0 & - & - & - \\
\hline PLANEJAMENTO & 37 & 37 & 26 & 100 & 72 & 26 \\
\hline EXECUÇÃO & 29 & 42 & 29 & 100 & 80 & 43 \\
\hline $\begin{array}{l}\text { MONITORAMENTO E } \\
\text { CONTROLE }\end{array}$ & 29 & 50 & 21 & 100 & 88 & 21 \\
\hline ENCERRAMENTO & 50 & 50 & 0 & 100 & 50 & - \\
\hline
\end{tabular}

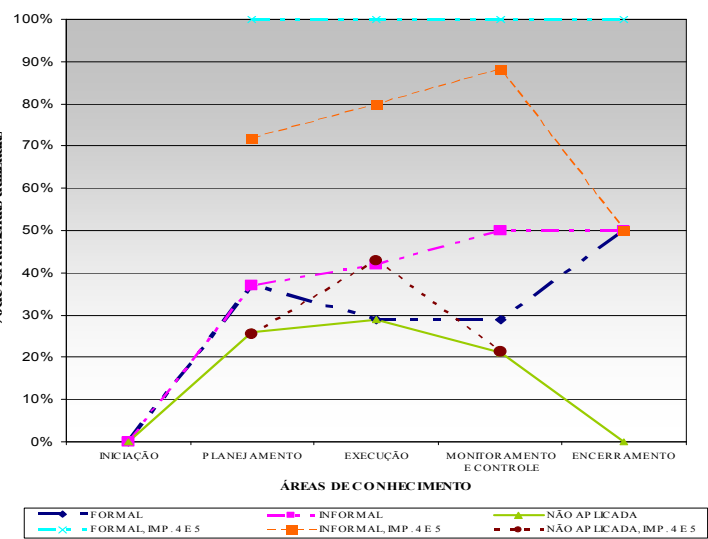

Figura 8 Utilização das ferramentas por grupos de processo 
Da Figura 9, alguns pontos que podem ser destacados são: 1) a área de riscos é a que apresentou o maior número de ferramentas informais com importância 4 ou $5(100 \%)$, seguida das áreas de tempo e comunicação (86\%), recursos humanos $(83 \%)$ e escopo e custo $(80 \%) ; 2)$ todas as ferramentas utilizadas formalmente no processo de desenvolvimento foram consideradas de importância 4 ou $5 ; 3$ ) todas as ferramentas que não são utilizadas na área de tempos e recursos humanos foram consideradas de importância 4 ou 5 , enquanto que $57 \%$ da área de riscos e $50 \%$ da área de custos; 4) a área que apresentou o maior número de ferramentas que não são utilizadas foi a área de qualidade (64\%), seguida da área de riscos $(44 \%)$.

\begin{tabular}{|c|c|c|c|c|c|c|}
\hline \multirow[b]{2}{*}{$\begin{array}{l}\text { ÁREAS DE } \\
\text { CONHECIMENTO } \\
\end{array}$} & \multicolumn{6}{|c|}{ \% DAS FERRAMENTAS UTILIZADAS } \\
\hline & 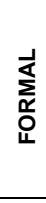 & 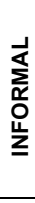 & 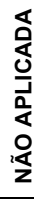 & 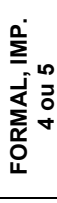 & 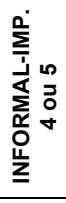 & 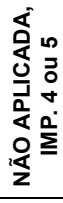 \\
\hline INTEGRAÇ̃̃O & 71 & 29 & 0 & 100 & 50 & - \\
\hline ESCOPO & 33 & 56 & 11 & 100 & 80 & 0 \\
\hline TEMPO & 50 & 44 & 6 & 100 & 86 & 100 \\
\hline CUSTO & 42 & 42 & 17 & 100 & 80 & 50 \\
\hline QUALIDADE & 0 & 36 & 64 & - & 50 & 14 \\
\hline $\begin{array}{l}\text { RECURSOS } \\
\text { HUMANOS } \\
\end{array}$ & 22 & 67 & 11 & 100 & 83 & 100 \\
\hline COMUNICAÇÃO & 36 & 64 & 0 & 100 & 86 & \\
\hline RISCOS & 13 & 44 & 44 & 100 & 100 & 57 \\
\hline
\end{tabular}

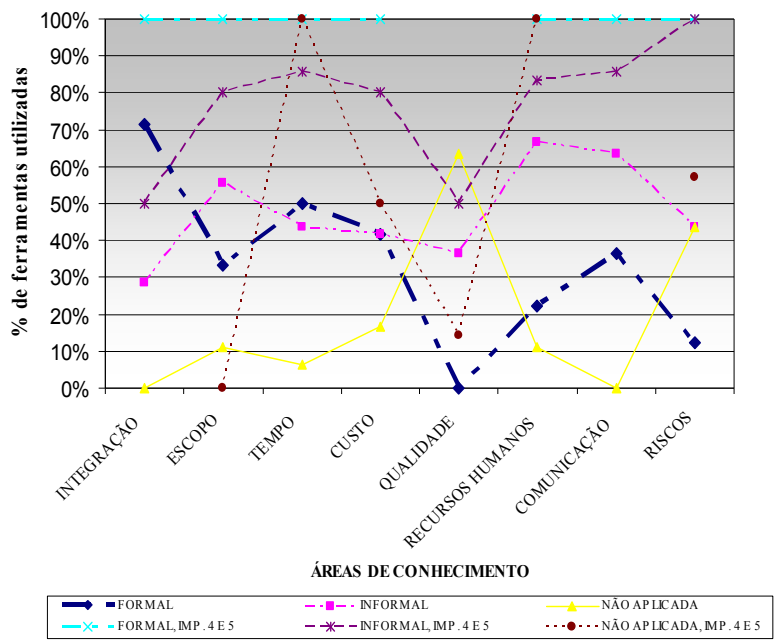

Figura 9 Utilização das ferramentas por grupos área de conhecimento

\section{Conclusão}

O presente trabalho apresentou um estudo inicial sobre alguns aspectos de como se daria a aplicação do gerenciamento de projetos no processo de desenvolvimento de produtos. O presente artigo apresenta uma visão geral do processo de desenvolvimento de produto e o situa nos processos de gerenciamento de projetos. Este seria um bom início para estudar a dinâmica desse processo e de como práticas reconhecidas de gerenciamento de projetos podem contribuir para o bom desempenho do processo de desenvolvimento.

Do estudo de caso realizado, pode-se concluir que apesar de grande parte das ferramentas citadas serem usadas para gerenciamento do desenvolvimento ( $76 \%$ das ferramentas citadas), menos da metade (34\%) são utilizadas de maneira formal, o que pode acarretar em falta de um padrão para o gerenciamento e falta de entendimento comum. Esse fato pode ser justificado devido ao elevado número de projetos sendo gerenciados simultaneamente e/ou por se tratarem de projetos incrementais, que são projetos mais simples.

O fato de $79 \%$ das ferramentas que são aplicadas de maneira informal terem importância 4 ou 5 , pode sugerir a necessidade de se formalizar o processo de gestão do desenvolvimento. Porém, não é possível concluir isso, pois, como mencionado anteriormente, isso pode ser devido aos projetos serem caracterizados como incrementais (e contemplar menos fases e atividades) e não requerer a aplicação de algumas ferramentas.

Os dados e as informações obtidas na realização do estudo de caso permitem afirmar que, apesar de as ferramentas de gerenciamento de projeto estarem bastante difundidas, elas ainda não estão sistematizadas nos processos de desenvolvimento das unidades de estudo. Não se pode, porém, afirmar o mesmo para toda a empresa, pois, conforme mencionado anteriormente, os desenvolvimentos realizados nessas unidades se caracterizam por projetos incrementais e os produtos das unidades investigadas se encontram em estágios avançados do ciclo de vida. 
Os resultados mostram também que o maior número de ferramentas está sendo aplicado no grupo de processos de planejamento e, em seguida, no grupo de monitoramento e controle. Da mesma forma, a área que apresenta o maior número de ferramentas utilizadas é a área de tempo, seguida das áreas de comunicação, custo e riscos.

O grupo que apresenta o maior número de ferramentas com importância 4 ou 5 é o grupo de monitoramento e controle, seguido dos grupos de execução e planejamento, o que pode sugerir pontos onde o processo pode ser formalizado. Já o grupo de execução apresenta o maior número de ferramentas que não são utilizadas no processo, seguido pelo grupo de planejamento e monitoramento e controle. Esse fato pode sugerir a implementação dessas ferramentas no processo de gerenciamento do desenvolvimento.

De forma similar, uma das áreas que apresentou maior deficiência foi a área de riscos, onde $44 \%$ das ferramentas são utilizadas de maneira informal (e apresentam uma importância de 4 ou 5) e 44\% das ferramentas apresentadas nesse trabalho para esta área não são utilizadas.

Com base nos dados da pesquisa, mesmo que as ferramentas e técnicas não sejam utilizadas de maneira sistêmica e formal, pode-se concluir que elas são aplicáveis ao processo de desenvolvimento de produto e auxiliam o seu gerenciamento, uma vez que $77 \%$ dessas ferramentas foram consideradas de importância 4 ou 5. Porém, até mesmo como proposta para pesquisa futura, a forma com que ela será aplicada durante o processo de desenvolvimento precisa ser mais bem estudada, atentando-se para os detalhes das atividades de desenvolvimento e as interações existentes nesse processo. Essa é uma possibilidade para continuidade desse trabalho.

\section{Referências}

CHEN, C. H.; LING, S. F.; CHEN, W. Project scheduling for collaborative product development using DSM. International Journal of Project Management, v. 21, n 4, p. 291-299, 2003

COOPER, R.G.; KLEINSCHIMDT, E.J. Benchmarking the firms critical factor in new product development. Journal of Product Innovation Management, v. 12, n 5, p. 371-391, 1995.

COOPER, R. G. Winning at New Products - Accelerating the Process from Idea to Lauch. 3ed. Cambridge, Massachusetts: Perseus Publishing, 2001.

ENGWALL, M.; KLING, R.; WERR, A. Models in action: how management models are interpreted in new product development. $R \& D$ Management, v. 35, no. 4, p. 427-439, 2005.

FORMÁGgIO, I. A. ; MIGUEL, P. A. C. . Estudo Inicial sobre a Utilização do QFD como Ferramenta Estratégica no Planejamento de Novos Produtos. In: XXII ENCONTRO NACIONAL DE ENGENHARIA DE PRODUÇÃO, Curitiba, v. I, p. 121, 2002.

FORMOSO, C. T.; TZORTZOPOULOS, P; LIEDTKE, R. A model for managing the product development process in house building. Engineering, Construction and Architectural Management, v. $9, n^{\circ} 5 / 6$, p. 419-432, 2002.

GRANT, K; PENNYPACKER, J. S. Project Management Maturity: An Assessment of Project Management Capabilities Among and Between Selected Industries. IEEE Transactions on Engineering Management, v. 53, nº 1, feb. 2006.

KERZNER, H. Project Management - A Systems approach to Planning, Scheduling and Controlling, $7^{\circ}$ ed., New York, John Wiley \& Sons. Inc., 2001. 
PATAH, L. A.; CARVALHO, M. M. Strategic alignment of project management office: na analysis of multiple cases. In: EurOMA International Conference on Operations and Global Competitiveness, Budapest, 2005.

PMI. PROJECT MANAGEMENT INSTITUTE. A Guide to the Project Management Body of Knowledge (PMBoK Guide), 2004.

ROZENFELD, H.; FORCELLINI, F. A.; AMARAL, D. C.; TOLEDO, J. C. D.; SILVA, S. L. D.; ALLIPRANDINI, D. H.; SCALICE, R. K. Gestão do Desenvolvimento de Produtos - Uma referência para a melhoria do processo. São Paulo: Saraiva, 2006.

SÖDERLUND, J. Managing complex development projects: arenas, knowledge processes and time. $R \& D$ Management, v.32, $\mathrm{n}^{\circ}$ 5, p.419-430, 2002.

ULRICH, K.; EPPINGER, S. Product Design and Development. New York: McGrawn-Hill, 1995.

YIN, R. K. Case study research: design and methods. New Delhi: Sage, 1984. 